\title{
Perioperative risk stratification in cardiac patients undergoing noncardiac surgery
}

Lee Goldman MD

\section{Objectives}

Review of the use of clinical data to assess the cardiac risk of noncardiac surgery and present the latest data and recommendations regarding the value, or lack thereof, of preoperative, noninvasive testing and therapeutic interventions, including beta-blockers and coronary revascularization.

A handout will be provided at the meeting. 